Int. J. Dev. Biol. 49: 131-136 (2005)

doi: $10.1387 / \mathrm{ijdb} .041965 \mathrm{ac}$

\title{
The Le Douarin phenomenon: a shift in the paradigm of developmental self-tolerance
}

\author{
ANTÓNIO COUTINHO* \\ Laboratoire Européen Associé au CNRS, Instituto Gulbenkian de Ciência, Oeiras, Portugal.
}

\begin{abstract}
Ever since the foundations of Immunology, "self-tolerance" has remained a central issue in this field, pertaining to basic and clinical questions alike. Burnet and Medawar shared the Nobel Prize in 1960 for proposing that tolerance is induced by tissue antigens, if present during the development of the immune system during the embryonic/neonatal period. Very elegant experiments by Le Douarin and colleagues in the 1980's demonstrated that this is not the case; rather, the establishment of tolerance to peripheral tissues requires thymic epithelium which selects CD4 T lymphocytes mediating "dominant tolerance". The recent wealth of work on "regulatory T cells", as well as observations on the selective regulation of "tissue-specific" gene expression in thymic epithelial cells, confirm the critical relevance of those seminal findings in modern immunology.
\end{abstract}

KEY WORDS: immunological tolerance, embryonic graft, thymic epithelium, regulatory T cell

With few exceptions (e.g. Vaz and Varela, 1978), immunologists claim that the «self-nonself discrimination» (SNSD) is the essential core of their preoccupations. The biological problem is indeed tremendous and it has been at the heart of the discipline, nearly from its very beginning. Thus, to ensure defense against infectious agents which can very rapidly evolve their antigenicity, the immune system must be endowed with a «complete repertoire» of specificities (Coutinho, 1980), that is, with an «open-ended» competence to recognize all possible molecular shapes. Furthermore, specific recognition must both be «coupled» to ridding mechanisms, which eliminate the microbe and neutralize its toxins, as well as to the establishment and maintenance of «memory», which provides selective immunity to the survivors of a primary infection. On the other hand, such universal recognition and effector mechanisms must not result in «ridding» responses against «self» antigens, thus raising the fundamental question of the molecular and cellular mechanisms involved in self-tolerance. Given that specific recognition is essentially derived from random genetic processes that operate somatically (Tonegawa, 1983), how is it possible that the vertebrate immune system recognizes and eliminates all antigens, except those pertaining to the organism itself?

Beyond its intrinsic difficulty, the problem and related debates have been colored by «personal»notes, actually from its inception. Paul Ehrlich declared autoantibody production to be «dysteleological» already in 1902, fueling an intense scientific dispute between the German and the French (Russian) schools. Times were ripe for that: Pasteur had just refused a Honoris causa degree from the University of Bonn, while Ehrlich's humoral and
Metchnikoff's cellular theories of immunity were starting to fight their way quite strongly. As Ehrlich denied the existence of autoantibodies, Metchnikoff's students at the Pasteur Institute (mainly Besredka and Metalnikoff) saw the possibility of proving him wrong, what they did in a most forceful manner, by demonstrating the production of autoantibodies against a variety of tissue antigens. With the predominance of the German school, however, the persistent paradigm was that of Ehrlich's, namely that autoantibodies simply could not (and did not) exist. This had serious consequences for the development of the discipline. Thus, for another 50 years or so, diseases caused by autoantibodies were not recognized, in spite of the very early demonstration of their existence. As Silverstein pointed out, this founding influence was so strong that excellent scientists «refused» to see their own evidence in this respect (Silverstein, 1979). Interestingly, the controversy persisted even after the association of autoantibodies with autoimmune diseases became generally accepted. More than 100 years after Ehrlich, current immunological theory continues to host views that claim the principal inability of healthy individuals to produce autoantibodies (Langman and Cohn, 2000), side by side with other theories defending that autoantibody production is a central component of immune physiology (Coutinho, 1995). I would think that there is no need to insist that «natural tolerance» and SNSD is the most immunological of all problems. After Jerne introduced Darwinian principles in the field (Jerne, 1955), Burnet invented the «clonal

Abbreviations used in this paper: SNSD, self-nonself discrimination.

\footnotetext{
*Address correspondence to: Dr. Antonio Coutinho. Instituto Gulbenkian de Ciência, Rua da Quinta Grande, 6, Apart. 14, 2781-901 Oeiras, Portugal. Fax: +351-21-440-7970. e-mail: coutinho@mail.igc.gulbenkian.pt
} 
selection theory» (Burnet, 1957) and Tonegawa solved the riddle of «too many antibodies for just a few genes» (Tonegawa, 1983), the issue of tolerance became, in the opinion of many, the last question in the field.

\section{Burnet's developmental tolerance hypothesis and Medawar's experiments: learning «self» by negative selection}

Already in the original paper of his general theory, Burnet had «solved» the problem of self-tolerance, by taking up Ehrlich's principle and framing it within «clonal selection». Thus, with two relatively simple assumptions, Burnet provided the first hypothesis of "developmental tolerance» and a coherent framework to approach the question: (1) all immunocompetent cells are produced in the embryo, that is, during the time when they are exclusively exposed to "self» antigens; (2) there is a developmental program determining that antigen recognition kills the specific cells in embryonic life, while it stimulates them after birth (when «nonself» antigens are also present in the organism). Clearly, such a scheme satisfies to the Ehrlichian principle of no recognition of «self», while allowing for responses to «nonself». The theory suggested that natural tolerance requires somatic «learning of self» and that such learning is developmentally controlled, taking place exclusively in the embryo. This seemed all the more necessary, as antigenic polymorphisms in the species could not all be genetically linked to lymphocyte recognition receptors, making it obvious that «self» had to be learned anew by the developing immune system from what is present in the embryo. The embryonic advantage owes to its seclusion from «nonself» antigens, allowing for a «safe» screen and elimination of the relevant clones («negative selection», in the language of today). As to the developmental shift in the fate of specific cells after antigen encounter, many a mechanism could be thought to ensure it and this would be certainly discovered sooner or later.

Burnet and Medawar shared the Nobel Prize in 1960, precisely for their «discovery of acquired immunological tolerance». Medawar and co-workers invented an experimental system in mice, which provided the means to analyze earlier observations by Owen in embryonic tolerance. Owen had noted that dizygotic twin calves, which had shared placenta during embryonic life, were chimeric for blood cells in adult life and, thus, tolerant to each other's tissue antigens. It would appear that each of the tolerant twin calves, aided by a mixed blood circulation, had also «learned» as "self» the twin's antigens to which they were exposed as embryos. Medawar then demonstrated that inbred mice, which were given at birth spleen and bone marrow cells from $F 1$ semi-allogeneic donors, would aquire life-long tolerance to skin grafts of the other parental strain, while normally rejecting third-party donor grafts (Billingham, 1953). "Learning of self» had found a sound experimental demonstration, which came to comfort theoretical expectations. Thus, Medawar's experimental demonstration of the specificity of «tolerance» was compatible with clonal selection's central rule, distributing different reactivities by independent clones of immunocompetent cells. In addition, Medawar and colleagues demonstrated that the period of susceptibility to tolerance induction was rapidly closed after birth, supporting Burnet's assumption that tolerance was exclusively acquired in the embryo. In spite of Medawar's own findings that only hematopoietic cells were «tolerogenic», these very attractive notions were unwarrantedly extended to all antigens present in the embryo. Irrespective of the detailed mechanisms involved, it became generally accepted that the «immunological self» is defined by learning the antigenic composition of the body during the embryonic and perinatal life: «self» is «what is there in development», regardless of its cellular nature.

\section{Le Douarin's experiments on tolerance: embryonic tissues are not tolerogenic in embryos!}

As natural tolerance is acquired in the embryo, SNSD is a question that brings together immunology and development. Not surprisingly therefore, the modern solution to the question was produced in Nogent, a laboratory that excelled in both fields. Interestingly, however, the original observations were carried out in chickens and quails, an experimental system that is far from the daily concerns of todays' immunologists. This might well explain why it has taken long for the immunological community to understand their crucial importance.

The series of experiments which drastically changed the way of thinking about natural tolerance by the end of the 1980's are of an extreme simplicity and elegance, if difficult to perform. The first experiments consisted in grafting embryonic tissues from quails into age-matched chick embryos and following their performance and survival and birth and in adult life. Most surprisingly for all of us immunologists, perhaps even for Le Douarin and colleagues, the grafts were well accepted and fully functional in the newly hatched birds, only to be acutely rejected a few weeks later, at the age of acquisition of immunocompetence. The observations were all the more relevant and surprising, as the grafts were performed very early in embryonic life, at a stage when no lymphoid development had yet occurred. As seen above, Medawar's experiments had shown that preparations from liver, muscle and skin were unable to induce tolerance to skin grafts in adult life, in clear contrast with hemopoietic (spleen and bone marrow) cells. In this system, however, the «tolerogenic regimen» was administered to mice at birth, well after lymphocytes had been produced. Furthermore, as it turned out, there was some strain variation in the tolerogenic competence of hemopoietic cells themselves when given at birth, some mouse strains requiring intra-uterine injections for full tolerance, others being permissive for several days after birth. In other words, the predominant notion was that of a quantitative difference between various cell types in their ability to induce tolerance, hemopoietic cells being the most «tolerogenic». This might explain why many recurrent analyses of the "Medawar system» were limited to the injection of hemopoietic cells and why current thinking retained the tolerogenic potential of hemopoietic cells, but not the failure of other cell types.

Le Douarin's experiments made it clear that embryonic rudiments of several organs and tissues (for example, limbs, bursa and brain) were surprinsingly unable to tolerize recipients at pre-lymphoid stages. After all, «self is not all that is there».

This was the first step of the Nogent's group, along the way to change the paradigm of developmental tolerance. The difficulty of conventional immunology to accept it was an excellent token of how revolutionary these results were. I am convinced that every immunologist, Burnet to start with, would have predicted that embryonic grafts would be «tolerated» for life, according to the 
central «dogma» of tolerance and learning of «self» during a critical developmental period. There was no reason, therefore, to expect that such grafts would not be fully tolerated, since they were present in the recipient embryos, just as if they were part of their own bodies. I vividly remember a conference, by the late 1980's at which Nicole Le Douarin discussed some of her results on the fate of embryonic grafts. I gave the next talk, with the rather conventional view that «self is not a genetic or biochemical listing» and it simply represents "what is there in development». Gunther Stent, who was chairing the session, pointed out to me that this view was simply not compatible with Le Douarin's observations and that immunologists like me should rather reflect on such results and elaborate on a suitable explanation.

\section{Lederberg's alternative for recessive tolerance did not hold either}

Regardless of the cell types that are endowed with tolerogenic competence, Medawar's experiments had demonstrated that acquisition of tolerance is restricted to embryonic and peri-natal times. Burnet's simple postulates on natural tolerance were thus comforted. Unfortunately, however, the vertebrate immune system, particularly that of mammals, is far more «dynamic» than Burnet could have anticipated. Thus, soon thereafter, it became clear that immunocompetent cells are lymphocytes and that new lymphocytes are produced throughout life, excluding the beautiful simplicity of Burnet's developmental scheme. There is, after all, no developmental coincidence between the period of tolerance acquisition and that of lymphocyte production, such that, throughout life, newly produced lymphocytes are not secluded from «nonself» antigens. Hence, SNSD must owe to mechanisms other than those suggested by Burnet. The central notion of "self learning by negative selection» was soon rescued, however. As early as 1959, Lederberg postulated that, whenever produced, lymphocytes go through a period of «immaturity» during which antigen recognition results in their death; only after further «maturation», lymphocytes respond to antigens by activation and differentiation to effector functions (Lederberg, 1959). As lymphocytes develop in the presence of "self» antigens in the bone marrow and thymus, tolerance would be established by deleting self-reactive cells at this early stage, contrasting with those lymphocytes directed to «nonself» antigens, which would only encounter antigens in the peripheral organs, once they were fully «mature».

Attractive as it might have been, Lederberg's hypothesis, which was to provide the central conceptual framework in SNSD for the next 40 years, «transferred» the developmental issue in tolerance from the whole organism to single cells! Beyond this epistemological draw-back, the hypothesis does not account for the observations that tolerance acquisition is restricted to the developmental time of the organism, not to that of single cells. Lymphocytes traverse similar stages of immaturity in the embryo (when tolerance operates) and in the adult (when it does not). Surprisingly, current immunological beliefs have kept both Lederberg's hypothesis and Medawar's findings and very few immunologists have been concerned with the ensuing (and rather obvious) «time problem»!

For several decades, the issue was increasingly confused by the variable criteria used to define a state of tolerance. In many cases, the inability to score a response in a particular test system, often in vitro, would be designated as «tolerance». Not surprisingly, therefore, many publications reported on the induction of tolerance to various antigens in adult life. Yet and in spite of much work in transplantation tolerance, surgeons continued to face the proverbial difficulties with organ transplantation, demonstrating, if in a negative manner, that acquisition of tolerance is a developmental problem indeed. With time, Medawar's observations seemed forgotten, allowing for the progressive installation of the «Lederberg's solution». In the early 1980's, rapid progress of cellular and molecular immunology, lead to the identification of $T$ cell receptors and specific antibodies were produced to «families» of such receptors, making it possible to follow «clones» of cells from their production in the central organs to the periphery. This was also greatly facilitated by the technique of generating transgenic mouse lines, in which most $\mathrm{T}$ or $\mathrm{B}$ lymphocytes would express a single clonal receptor, chosen a priori by the investigator. Much of the two following decades were devoted to the «discovery» and the many times repeated experimental confirmation of Lederberg's hypothesis. Developing self-reactive lymphocytes in thymus and bone marrow were deleted as predicted. Yet, deletion was rarely or never complete, raising pertinent questions on the hypothesis, all the more so given the immune system's ability to expand clones that are present at very low frequencies. Moreover, the predicted correlation between lymphocyte deletion and tolerance did not seem to hold, as defects in deletion could neither be demonstrated in autoimmune conditions, nor give rise to them. By reading the litterature, however, it did seem that the problem was exhaustively solved: tolerance was claimed to be undoubtedly acquired by «negative selection» of specific self-reactive cells along with the process of their production. Whenever clonal elimination could not be detected, some kind of in vitro assay would show «functional deletion» or «anergy» of the specific cells, or else, «peripheral failsafe mechanisms» would be postulated.

Concerning $T$ lymphocytes, the notion of «negative» selection was re-inforced by the previously established notion of thymic selection, required for MHC-restriction. All these notions also gained strength with the discovery of programmed cell death in lymphocytes and with the «division of labor» proposed for the two types of thymic stromal cells: radio-resistant epithelial cells were responsible for «positive selection» in the cortex (as demonstrated early-on for MHC-restriction), while hematopoietic antigen-presenting cells would mediate negative selection in the medulla bringing back Medawar's observations and a «deletional» explanation. All along the 1990's, it was taken as established that «the thymus selects the useful, neglects the useless and destroys the harmful» (von Boehmer et al, 1989) the latter being all self-reactive lymphocytes, even if with minimal affinities. The equivalent process in bone marrow $B$ cell development had also made its way to explain B cell tolerance, in spite of the abundant observations revealing the presence of selfreactive antibodies in normal serum and the facility to demonstrate auto-reactive $B$ cell clones after polyclonal activation by mitogens. It was argued that these sets of data were due to «low affinity» crossreactivities and entirely irrelevant from the functional point of view. However, the very experiments in transgenic mice which provided the strongest support for deletional B cell tolerance, had shown precisely the opposite, namely that deletion operates below the affinity threshold required for detection of antibody reactivity. Surprisingly again, the experimental systems that produced such demonstrations of deletional tolerance in antibodies had forced the expression in developing bone marrow $B$ cells of highly mutated 
antibody receptors that could never occur but in peripheral germinal centers! Nothing, however, seemed to be going wrong in the kingdom of Denmark. Yet, already then, a number of reasons suggested that the question was far from settled.

One of the difficulties with negative selection had to do with the extensive degeneracy of antigen recognition by $T$ and B lymphocytes, making it difficult to understand how deletion could remove all selfreactive cells leaving behind an immunocompetent repertoire. This was particularly critical, as it is very likely that antigen concentrations/ densities presented to differentiating lymphocytes in the central lymphoid organs are quite different from those encountered by mature cells in the periphery, often in conditions of inflammation, which enhance the expression of «accessory molecules» and the ability of lymphocytes to respond. The major problems with the idea, however, were the «space» and the «time» problems. Thus, for ensuring tolerance by negative selection, it would be necessary that every developing lymphocyte encounters every self-antigen along its development. In other words, it would be necessary that all relevant antigens in the body are present in thymus and bone marrow and, in addition, that all self-antigens would be expressed by the few «presenting cells» that each differentiating lymphocyte contacts before it completes maturation. The old «time problem» was, perhaps, even more difficult: since tolerance is established only in the embryo while new lymphocytes are produced throughout life in the presence of both self and non-self antigens, it would seem necessary that differentiating lymphocytes in the adult had access to some kind of memory of the «self» antigenic composition in development. This could obviously not be achieved by negative selection. Most surprisingly, or maybe not, the overwhelming majority of all experiments on «tolerance by deletion» were conducted in adult animals, when tolerance can no longer be acquired. Actually, it came as a surprise that neonates appeared quite refractory to thymic deletion, in conditions which would readily detect deletion in adult life.

In spite of the strength of the dogma, however, a few groups around the world kept some sort of passive resistance to the notion of clonal deletion as the basis for natural tolerance. These insisted that autoreactive $T$ and $B$ cells exist in normal, healthy individuals and some even spoke of «physiological autoreactivity», as they could demonstrate the «natural activation» of self-reactive lymphocytes in the absence of environmental stimulation. Some did go as far as arguing that physiological autoreactivity was actually necessary to prevent pathogenic autoimmune manifestations. This was suggested by the therapeutic effects for autoimmune patients of physiological autoantibodies contained in serum immunoglobulin preparations from healthy donors, as well as by the protective "vaccinating" effects of autoreactive T cells in autoimmune diseases. This small group of «opponents» to the simplistic solution of deletional tolerance, however, had little or no impact in the current thinking

\section{Le Douarin's results established the role of thymic epithelium selection in dominant tolerance}

It was in this general context that Le Douarin and colleagues produced a second piece of evidence that forced a reappraisal of the whole framework, even if quite many years later. Using the same experimental system of embryonic grafts, the group at Nogent demonstrated that transplantation of thymic rudiments, before colonization by hemopoietic precursors, would establish life-long tolerance to all grafts of peripheral organs and tissues from the same donor (Ohki et al, 1987). In short, Le Douarin demonstrated that peripheral, «tissue-specific» tolerance is centrally established by «pure» thymic epithelium. In addition to demonstrating that all kinds of peripheral tissues were not tolerogenic in the embryo, Le Douarin and colleagues thus showed that thymic epithelium had a full tolerogenic potential, matched only by the classical observations of Medawar for hemopoietic cells. Moreover, their elegant experimental system allowed them to exclude the participation of the latter, thus establishing that only two tissues in the body are actually capable of inducing (self-) tolerance. Finally and most intriguingly, tolerance established by a single tissue type, seemed to apply to all other tissues in the body, again, as in Medawar's experiments (Ohki et al., 1988). In my opinion, this set of observations is of comparable importance to that of Medawar and is endowed with a higher heuristic value for the solution of developmental tolerance, as they were to play a major influence in the conceptual shift that took place some 10 years later.

Even for «deletioners», thymic epithelium was not considered to excel in «negative selection» of developing T lymphocytes, such that Le Douarin's observations were likely due to an alternative mechanism. In the following few years, Le Douarin and colleagues provided the formal demonstration that TE-tolerance is, instead, "dominant» and mediated by self-reactive suppressive cells (Coutinho etal., 1993; Le Douarin et al., 1996). First, they adapted the experimental system to mice, where inbred strains and reagents provided for a detailed analysis of the respective cellular mechanisms. Rudiments of «pure TE», collected prior to hemopoietic colonization from 10 days-old embryos, were transplanted to newborn athymic mice, which were then tested in adult life by various tissue grafts. Using allogeneic TE/ recipient combinations, Le Douarin's group could show that tolerance was established to skin, thyroid and heart grafts of the TE donor type, by a mechanism which did not involve significant deletion or functional inactivation of specific T lymphocytes (Salaün et al., 1990). Thus, tolerant mice contained frequencies of graft-reactive T cells that were comparable to those in control, non-tolerant animals and these cells were fully functional. Moreover, in very elegant experiments involving T cell transfers from tolerant donors to syngeneic, athymic recipients, they demonstrated that tolerant donors contained $T$ cells that were perfectly capable of rejecting the tolerated grafts, but only after they had been removed from the "regulatory» influence of other T cells (Modigliani et al., 1995). Dominant tolerance, mediated by $\mathrm{T}$ lymphocytes that were specifically selected on thymic epithelium, had been established for the first time.

Le Douarin and coworkers did go further in the identification of the «regulatory cells» as CD4 T lymphocytes and in showing the ability of such cells to «educate», together with the specific tissue antigens, newly formed peripheral $T$ cells for similar regulatory functions (Modigliani et al., 1996). Finally, regarding cellular mechanisms of «regulation», they also came to several important conclusions: (1) newly formed $T$ cells could be peripherally educated, by thymusderived regulatory cells and antigen, to «perpetuate» antigen-specific dominant tolerance, much in the way that Waldman designated as «infectious tolerance» in his system (Waldmann and Cobbold, 1998); (2) mature T cells that had resided in the periphery for longer periods of time could only be «suppressed» by regulatory cells and apparently remained as «naîve» cells after exposure to antigen; (3) antigen-primed T cells were resistant to «regulation», a finding that might be relevant when considering the potential use of regulatory $T$ cells and their manipulation in the therapy of autoimmune diseases. 
Le Douarin's results were of crucial relevance for the establishment of a coherent framework in «dominant tolerance», as they provided the developmental and physiological "sense» that organised and consolidated several other sets of observations in autoimmune diseases and transplantation tolerance in adults. «Non-believers» in tolerance by negative selection often pointed out that there was no correlation between defects in deletion and autoimmunity, which was, instead, surprisingly manifested in conditions of lymphocyte depletion. The experiments of Mason and colleagues (Fowell and Mason, 1993) and those of Sakaguchi (Sakaguchi et al., 1995) were particularly striking: they showed that removal of some subsets of $T$ cells, either by early thymectomy or by cell fractionation followed by transfers to «empty» hosts, would often result in autoimmune manifestations. These observations demonstrated two critical points: first, those normal healthy donors contain self-reactive T cells that are capable of inducing autoimmune disease in lymphopenic hosts; second, that healthy animals also contain subsets of T lymphocytes that inhibit the activity of pathogenic T cells co-existing in the animal. One other set of experiments came to support such interpretations, if out of the frame of physiology and development. These were the observations from Waldman and colleagues that animals could be tolerized to allogeneic grafts as adults, if «under cover» of massive infusions of anti-CD4 antibodies (Waldmann and Cobbold, 1998). Thus, such tolerant animals contained a class of T lymphocytes that could transfer the tolerant state to naîve recipients! A few years later, the small community of «dominant tolerance» groups welcomed the most elegant experiments of Lafaille and Tonegawa (Lafaille et al., 1994). Having produced a transgenic mouse in which most CD4 T cells expressed an autoreactive receptor isolated from an encephalitogenic clone, they found, as others before, that the presence of very large numbers of potentially pathogenic $T$ cells did not result in autoimmune disease (as it would have been expected by the dominant theory). In contrast with others, however, they had the insight of crossing the transgenic mice to a Rag-deficient «background», generating conditions which excluded the presence of any other lymphocytes but the transgenic autoreactive cells. The results could not be more clear and striking: $100 \%$ of these animals developed «spontaneous» and rapidly fatal autoimmune encephalitis, which could be prevented by transferring low numbers of CD4 T cells from normal syngeneic donors. These experiments established, for the first time, that autoimmmune disease does not correlate with the presence (even in very large numbers) of pathogenic autoreactive $T$ cells, but with the absence of «regulatory T cells» (even if in very small numbers).

Scientists generally take very progressive positions in world matters, but are often very conservative when it comes to their own convictions. In spite of this wealth of concordant results, in spite of Le Douarin's observations which prompted developmental models of regulatory $T$ cell selection and «dominant tolerance», «recessive tolerance» by deletion of autoreactive cells continued to represent the most prominent (the only allowed?) theoretical framework in the field. Furthermore, the essential arguments were progressively substituted by findings and discussions which did not address SNSD at all, but mediated notions of exogenous «induction» of (or «protection» from) autoimmunity. The «danger hypothesis» that Matzinger built upon the late 1980's revival of «innate immunity», claimed that clonally specific SNSD did simply not exist and joined the popular belief that all autoimmunity merely represented «immunopathology» provoked by responses to infectious non-self.
On the other hand, many believed that the Th1/Th2 dichotomy of CD4 T cells and their mutual suppression provided the solution to natural tolerance and autoimmmunity, again out of context with SNSD. In short, the common conviction was that infection-dependent immunopathology would be «suppressed» via the Th1/Th2 pathway, failing to address the root of the question, i.e., how class-regulation was developmentally coupled to specific clonal repertoires.

It took nearly one decade for the appropriate recognition of all those results on «dominant tolerance», Le Douarin's included. Thus, only by the 2001 International Immunology Congress in Stockholm, did «dominant» and «recessive» tolerance reach a quasi balanced representation in symposium lectures and workshops. By then, however, the wind had turned and many a believer in deletion had jumped the fence and was doing experiments on regulatory $T$ cells. Sadly, this evolution owed, perhaps more than to Le Douarin's experiments and to those of Mason, Sakaguchi, Waldman and Lafaille, to the availability of in vitro assays for «suppression», which allowed for the massive production of «suppressor» phenomenology. Meanwhile, however, two other important sets of in vivo observations had been reported. On the one hand, Kyewski's observations had imposed the notion that many «tissue-specific» antigens were actually available in the thymus, each expressed by a small cluster of a few TE cells (Derbinski et al., 2001). On the other hand, Caton and colleagues had shown that regulatory $\mathrm{T}$ cells are selected from differentiating CD4 lymphocytes expressing receptors with high affinity to auto-antigens selectively expressed by TE cells (Jordan et al., 2001)!

\section{How the framework changed after Le Douarin's experiments}

The outlook on natural tolerance is thus very different today, as compared to what it was some 10-15 years ago. If we are still far from a complete, perhaps even correct, understanding of the cellular and molecular mechanisms of natural tolerance, it is clear that the current framework has radically turned around. The field did move from a predominance of recessive tolerance achieved by «negative selection», to considering as a valid alternative that developmental tolerance is dominant and established by the "positive selection» and «natural activation» of self-reactive cells to a «regulatory» functional phenotype. It would actually seem that some general agreement has been reached: negative selection does operate in lymphocyte development, it is likely to contribute to the robustness of tolerant states, but alone is not sufficient to ensure natural tolerance. Accordingly, physiological auto-reactivity is now accepted, as least for certain types of lymphocytes (e.g., regulatory T cells, marginal zone B cells), as well as, in some types of conditions, for all of them (e.g., «homeostatic proliferation»). The general conceptual frame is thus in better agreement with essential characteristics of the vertebrate immune system: first, with the degeneracy of Variable-region recognition, antigen cross-reactivity and «multireactivity» of neonatal repertoires; second, with «natural activation» of B and T lymphocytes in normal animals, even if secluded from environmental stimulation; third, with the fact that immune responses are just not the sum of independent clonal activities, but are determined by mutual functional interactions amongst activated cells; finally, with the relevance of «class regulation» of lymphocyte responses in determining between tolerance and the various types of immune responses. The basis of SNSD is not a matter of recognition repertoires, but lies on the 
«coupling» of specific recognition to effector functions, such that tolerance is but one form of response coupled to a non-ridding function that is selected in development. More importantly, the notion of «dominant tolerance» does account better for the «space» and the «time» problems of developmental tolerance, as it allows for building up a memory of developmental self, maintained throughout life and throughout the body by regulatory $T$ cells.

Interestingly, these notions have had important implications in medically relevant areas. First of all, in what concerns therapeutic strategies in autoimmune diseases. Clearly, within «recessive tolerance» models, «immunosuppression», aiming at eliminating auto-reactive lymphocytes, is the treatment of autoimmune diseases. Yet, using such strategy, there are no records of any patient who has ever been cured and all clinical benefits may be explained by the related anti-inflammatory effects of such treatments. In contrast, for "dominant tolerance», autoimmune disease results from deficits in numbers, functional competence, or specificity of autoreactive regulatory $T$ cells, such that autoimmune patients should be treated by immunostimulation, rather than by immunosuppression. This turn-around continues to pose daily problems to clinicians, but we all expect that progress in the pharmacology of regulatory $T$ cells will soon provide them with new tools for therapeutic intervention. Much needed novel strategies are also in development concerning transplantation tolerance. Furthermore, «dominant tolerance» notions have also open the way to critical discoveries in immunological conditions, other than autoimmunity and transplantation tolerance. Curroto de Lafaille and colleagues have shown that regulatory T cells play fundamental roles in inhibiting allergic responses and might have provided the key piece in this unsolved puzzle (Curroto de Lafaille etal., 2001). Now that intense efforts are devoted to establishing the genetic basis of autoimmune diseases and allergy, we can expect that data from physiopathology will complement results from basic research on natural tolerance and both will bring us closer to solving the medical problems. While the frequency of autoimmune diseases and allergies continues to increase in economically developed societies, we have no rational, specific or effective treatment for these conditions. Finally, we also have no effective vaccine against chronic infectious diseases, such as AIDS, malaria and tuberculosis. It would not be surprising that chronicity of infections is somehow related to the engagement of regulatory $T$ cells.

I have argued before that the culprit for this shocking lack of progress in «translational immunology» was «recessive tolerance». Would there be a grain of truth in this statement and the relevance of Le Douarin's work on tolerance would acquire yet further importance, now in applice.

\section{References}

BILLINGHAM, R. E., BRENT, L. and MEDAWAR, P. B. (1953). Actively acquired tolerance of foreign cells. Nature 172: 603-606.

BURNET, F. M. (1957). A modification of Jerne's theory of antibody production using the concept of clonal selection. The Australian J. 20: 67.

COUTINHO, A. (1980). The self-nonself discrimination and the nature and acquisition of the antibody repertoire. Ann. Immunol. 131D: 235-253.

COUTINHO, A., SALAÜN, J., CORBEL, C., BANDEIRA, A. and LE DOUARIN, N. (1993). The role of thymic epithelium in the establishment of transplantation tolerance. Immunol. Rev. 133: 225-240.
COUTINHO, A., KAZATCHKINE, M. D. and AVRAMEAS, S. (1995). Natural autoantibodies. Curr. Opin. Immunol. 7: 812-818.

CUROTTO DE LAFAILLE, M. A., MURIGLAN, S., SUNSHINE, M. J., LEI, Y., KUTCHUKHIDZE, N., FURTADO, G. C., WENSKY, A. K., OLIVARES VILLAGOMEZ, D. and LAFAILLE, J. J. (2001). Hyper immunoglobulin E response in mice with monoclonal populations of B and T lymphocytes. J. Exp. Med. 194: 1349-1359.

DERBINSKI, J., SCHULTE, A., KYEWSKI, B. and KLEIN, L. (2001). Promiscuous gene expression in medullary thymic epithelial cells mirrors the peripheral self. Nat. Immunol. 2: 1032-1039.

FOWELL, D. and MASON, D. (1993). Evidence that the T cell repertoire of normal rats contains cells with the potential to cause diabetes. Characterization of the CD4+ T cell subset that inhibits this autoimmune potential. J. Exp. Med. 177: 627-636

JORDAN, M. S., BOESTEANU, A., REED, A. J., PETRONE, A. L., HOLENBECK, A E., LERMAN, M. A., NAJI, A. and CATON, A. J. (2001). Thymic selection of CD4+CD25+ regulatory T cells induced by an agonist self-peptide. Nat. Immunol. 2: 301-306.

JERNE, N. (1955). The natural-selection theory of antibody formation. Proc. Natl. Acad. Sci. USA, $41: 849$.

LAFAILLE, J. J., NAGASHIMA, K., KATSUKI, M. and TONEGAWA, S. (1994). High incidence of spontaneous autoimmune encephalomyelitis in immunodeficient antimyelin basic protein T cell receptor transgenic mice. Cell 78: 399-408.

LANGMAN, R. E. and COHN, M. (2000). A minimal model for the self-nonself discrimination: a return to the basics. Semin. Immunol. 12: 189-195; discussion 257344.

LE DOUARIN, N., CORBEL, C., BANDEIRA, A., THOMAS-VASLIN, V., MODIGLIANI, Y., COUTINHO, A. and SALAUN, J. (1996). Evidence for a thymus-dependent form of tolerance that is not based on elimination or anergy of reactive T cells. Immunol. Rev. 149: 35-53.

LEDERBERG, J. (1959). Genes and antibodies - Do antigens bear instructions for antibody specificity or do they select cell lines that arise by mutation? Science 129: 1649-1653.

MODIGLIANI, Y., THOMAS-VASLIN, V., BANDEIRA, A., COLTEY, M., LE DOUARIN, N. M., COUTINHO, A. and SALAÜN, J. (1995). Lymphocytes selected in allogeneic thymic epithelium mediate dominant tolerance toward tissue grafts of the thymic epithelium haplotype. Proc. Natl. Acad. Sci. USA 92:7555-7559.

MODIGLIANI, Y., COUTINHO, A., PEREIRA, P., LEDOUARIN, N., THOMAS-VASLIN, V., BURLEN-DEFRANOUX, O., SALAUN, J. and BANDEIRA, A. (1996) Establishment of tissue-specific tolerance is driven by regulatory $T$ cells selected by thymic epithelium. Eur. J. Immunol. 26: 1807-1815

OHKI, H., MARTIN, C., CORBEL, C., COLTEY, M. and LE DOUARIN, N. M. (1987) Tolerance induced by thymic epithelial grafts in birds. Science. 237: 1032-1035.

OHKI, H., MARTIN, C., COLTEY, M. and LE DOUARIN, N. M. (1988). Implants of quail thymic epithelium generate permanent tolerance in embryonically constructed quail/chick chimeras. Development 104: 619-630.

SAKAGUCHI, S., SAKAGUCHI, N., ASANO, M., ITOH, M. and TODA, M. (1995). Immunologic self-tolerance maintained by activated T cells expressing IL-2 recepto alpha-chains (CD25). Breakdown of a single mechanism of self-tolerance causes various autoimmune diseases. J. Immunol. 155: 1151-1164.

SALAÜN, J., BANDEIRA, A., KHAZAAL, I., CALMAN, F., COLTEY, M., COUTINHO, A. and LEDOUARIN, N. M. (1990). Thymic epithelium tolerizes for histocompatibility antigens. Science 247: 1471-1474.

SILVERSTEIN, A.M. (1979). History of Immunology. Cell. Immunol., 42: 1-2.

TONEGAWA, S. (1983). Somatic generation of antibody diversity. Nature 302: 575 581.

VAZ, N. M. and VARELA, F. J. (1978). Self and non-sense: an organism-centered approach to immunology. Med. Hypotheses 4: 231-267.

VON BOEHMER, H., TEH, H. S. and KISIELOW, P. (1989). The thymus selects the useful, neglects the useless and destroys the harmful. Immunol. Today. 10: 57-61.

WALDMANN, H. and COBBOLD, S. (1998). How do monoclonal antibodies induce tolerance? A role for infectious tolerance? Annu. Rev. Immunol. 16: 619-644. 